\title{
The fauna of Sphaeroceridae (Diptera) in the Gemer area (Central Slovakia)
}

Jindřich Roháček

\begin{abstract}
The fauna of Sphaeroceridae (Diptera) in the Gemer area (Central Slovakia). - Čas. Slez. Muz. Opava (A): 60: 25-40, 2011.

Abstract: A synopsis of the fauna of the family Sphaeroceridae (Diptera) in the Gemer area (Slovakia) is presented. A total of 106 species are treated on the basis of previously published records and material examined. Each species is listed with comments about its general distribution, biology and faunistic and/or nature-conservancy importance. The regional fauna of Sphaeroceridae is distinguished not only for its high species diversity but also for numerous very rare, stenotopic, or endangered taxa. Among them, five species described from the area, viz. Elachisoma bajzae Papp, 1983, Herniosina pollex Roháček, 1993, H. horrida (Roháček, 1978), Opalimosina (Opalimosina) calcarifera (Roháček, 1975), Rachispoda segem (Roháček, 1991) and species hitherto known only from Gemer in Slovakia, viz. Crumomyia setitibialis (Spuler, 1925), C. zuskai (Roháček, 1976), Spelobia faeroensis (Deeming, 1966) and S. simplicipes (Duda, 1925), should be particularly mentioned. The peculiarities of the fauna of Sphaeroceridae in the Gemer territory are discussed including a review of species associated with cave habitats in the area and species most important from ecological, biogeographical and nature-conservancy points of view.
\end{abstract}

Key words: Diptera, Sphaeroceridae, faunistics, nature conservancy, Slovakia, Gemer

\section{Introduction}

The family Sphaeroceridae is one of the best studied families of Diptera in the Gemer area. The results of the author's collecting efforts in the Slovenský kras carst-region (undertaken in 1980-1982) and in the Muránska planina Mts (in 1982-1984), supplemented by some further material collected in the Gemer area by dipterists V. Zeman, S. Ošmera, R. Rozkošný and J. Máca, were published by J. Roháček and co-authors in various taxonomic and faunistic papers (Marshall \& Roháček 1984; Roháček 1975, 1977, 1978a,b, 1982, 1983a,b, 1991a,b, 1992, 1994a,b, 2001; Roháček \& Marshall 1982, 1986) and the majority of them summarized by Roháček (1986) in the faunal compendium Diptera Slovenska II (Diptera of Slovakia II) edited by the late J. Čepelák. Subsequently, additional faunal data were obtained by examination of interesting material of Sphaeroceridae collected in cave systems of the study area by V. Košel but these were only partly published (see Roháček 1991a, 1993; Roháček \& Košel 1993). New faunal data (including further species recorded from the Gemer territory) were obtained by examination of specimens of Sphaeroceridae collected recently in the study area by J. Ševčík in 2008-2010 (mainly from his Malaise traps in two localities of the Muránska planina Mts), new material from caves collected by V. Košel, A. Mock and L'. Kováč, a few specimens from a Malaise trap operated by L. Vidlička in Paseky nr. Tisovec in 2001, and a sample collected by the author in the Murán vicinity in 2010. This study of lesser dung flies belongs to a series of papers devoted to Diptera of the Gemer area (cf. Ševčík \& Kurina 2011; Roháček \& Ševčík 2011).

\section{Material and methods}

The present survey of species of Sphaeroceridae in the Gemer area is based on critical review of all published records supplemented by data obtained from the material collected recently in this territory, identified by the author and now mostly deposited in SMOC and in the collection of Dr. V. Košel (Bratislava). The nomenclature used here follows the most recent Checklist of Diptera of the Czech Republic and Slovakia, electronic version 2 (see Roháček 2009a). Species significant from a biogeographical, ecological or natureconservancy point of view are marked by “*” preceeding the species' name. 
Abbreviations used in text: MT = Malaise trap, $\mathrm{ST}=$ soil trap, $\mathrm{SMOC}=$ Slezské zemské muzeum, Opava, Czech Republic, VKB = collection of V. Košel, Bratislava, Slovakia.

\section{Study area}

The Gemer area is here understood as the territory of three national parks in central Slovakia - Muránska planina National Park (= NP), Slovenský raj NP and Slovenský kras NP. A part of the material presented in this study was collected within the framework of the All Taxa Biodiversity Inventories project (ATBI, see www.atbi.eu) organized by Workpackage 7 (WP7) of the "European Distributed Institute of Taxonomy" (EDIT, see www.e-taxonomy.eu). All three national parks represent well-preserved karst areas with both montane and thermophilous species included, including several endemic. The results of the species inventory within the ATBI project are available at http://www.atbi.eu/gemer/. Two Malaise traps were operated in the area - one at Fabova Hola Mt. in 2009 and the second in the Hrdzavá dolina valley in 2010. For more detail see Ševčík \& Kurina (2011).

\section{Synopsis of species}

\section{Copromyzinae}

\section{Alloborborus pallifrons (Fallén, 1820)}

Literature: Muráň-Hrdzavá dolina, Slovenský raj-sedlo Lipovec (Roháček 1986, 1992).

Remarks: A species restricted to the temperate and northern belt of the West Palaearctic area. It is a typical member of the community developing in horse dung on pastures.

\section{Copromyza equina Fallén, 1820}

Literature: Rožňava (Fritsch 1875, Thalhammer 1899), Slavec nr. Plešivec, Zádiel, Slovenský raj-sedlo Lipovec (Roháček 1986, 1989).

Remarks: A subcosmopolitan coprophagous species, usually living on dung in pastures but also on manure.

\section{*Copromyza montana Roháček, 1992}

Material: Muránska planina-Fabova hol’a res., MT, 15.v.-16.vi.2009, 4ð1우, J. Ševčík leg. (SMOC).

Remarks: An uncommon montane species associated with open (montane alpine meadows) and semi-open (thin montane forests) habitats. It has hitherto only been recorded from the Czech Republic, Germany, Italy and Slovakia (Roháček et al. 2001), in the latter country from the Nízke Tatry Mts, Belianske Tatry Mts and Nízke Beskydy Mts (Roháček 1992).

\section{Copromyza nigrina (Gimmerthal, 1847)}

Literature: Slovenský raj-sedlo Lipovec (Roháček 1986 as C. similis, Roháček 1989).

Remarks: A widespread, predominantly coprophagous, Palaearctic species, in Central and South Europe limited to montane altitudes.

\section{Copromyza stercoraria Fallén, 1820}

Material: Muránska planina-Fabova hol'a res., MT, 15.v.-16.vi.2009, 1ð1ㅜ, 16.vi.-25.viii.2009, 1ㅇ, J. Ševčík leg. (SMOC).

Remarks: A saprophagous Holarctic species, rather common in Slovakia. It is habitat- and altitude-tolerant and often occurs in burrows of small mammals. Interestingly not previously recorded from the Gemer area.

\section{Crumomyia fimetaria (Meigen, 1830)}

Literature: Muráň-Hrdzavá dolina, Zádiel (Roháček 1986, 1991a), Rožňava (Fritsch 1875, Thalhammer 1899, as Borborus).

Material: Muránska planina-Fabova hol’a res., MT, 16.vi.-25.viii.2009, 10^, J. Ševčík leg.; Muráň-Hrdzavá dolina, on decayed fungi, 7.x.2010, 1ठ1\%, J. Roháček leg. (SMOC); Slovenský kras-Májkova jaskyňa (cave), 1.viii.1990, 1ㅇ, V. Košel leg. (VKB).

Remarks: A common West Palaearctic species, frequently occurring in woodland habitats on decayed macrofungi.

\section{Crumomyia nigra (Meigen, 1830)}

Literature: Rožňava (Fritsch 1875, Thalhammer 1899, as Borborus), Zádielská planina (Roháček 1986), Zádiel, Slovenský kras-priepast' (chasm) Brázda, Slovenský raj-jaskyňa (cave) Komíny, Stratenská jaskyňa (cave) (Roháček 1991a),Vlčia jaskyňa (cave), Koniarova jaskyňa (cave) (Roháček 1991a; Roháček \& Košel 1993).

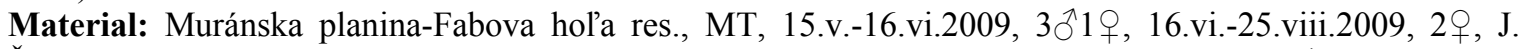
Ševčík leg. (SMOC); Slovenský raj-Medvedia jaskyňa (cave), 30.vii.1989, 20-25 m, 3ð̋2ㅇ, 25-30 m, 1ठำ 1 , 30-35 m, 1ठ, V. Košel leg. (VKB). 
Remarks: A Eurasian (but also known from Greenland) coprophagous species, common on dung in pastures.

\section{Crumomyia nitida (Meigen, 1830)}

Literature: Muráň-Hrdzavá dolina (Roháček 1986, 1991a), Slovenský kras-priepast’ (chasm) Brázda (Roháček 1991a), Vlčia jaskyňa (cave), Koniarova jaskyňa (cave) (Roháček 1991a; Roháček \& Košel 1993).

Material: Muránska planina-Fabova hol’a res., MT, 15.v.-16.vi.2009, 1ð2ㅇ, 16.vi.-25.viii.2009, $2 \precsim 2$, J. Ševčík leg.; Muráň-Hrdzavá dolina, MT, 1.v.-28.vi.2010, $2{ }^{\Uparrow} 1$ q, J. Ševčík leg. (SMOC); Slovenský krasjaskyňa (cave) Milada, 20.vi.2007, 5-6 m, 1q, 8.ix.2010, 5-6 m, 1ð̊, 10-12 m, 1ㅇ, V. Košel leg. (VKB).

Remarks: A common West Palaearctic species occurring in woods on wet rotting leaves along creeks and spring places.

\section{Crumomyia notabilis (Collin, 1902)}

Literature: Muránska planina-jaskyňa (cave) Stará Bobačka, Slovenský kras-Stará brzotínska jaskyňa (cave) (Roháček 1991a), Vlčia jaskyňa (cave), Koniarova jaskyňa (cave) (Roháček 1991a; Roháček \& Košel 1993).

Material: Muránska planina-Fabova hol'a res., MT, 15.v.-16.vi.2009, 1ð̋, J. Ševčík leg. (SMOC); Muránska planina-jaskyňa (cave) Teplica nr. Tisovec, 7.viii.1991, 1q; Slovenský kras-Hrušovská jaskyňa (cave),

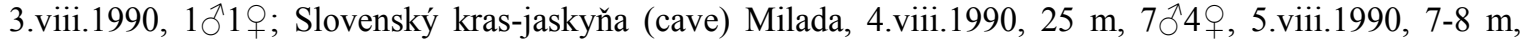

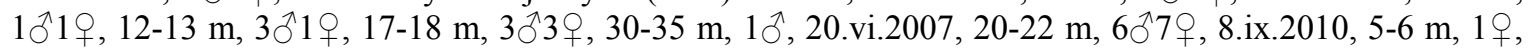
20-22 m, 4ð̄, V. Košel leg. (VKB).

Remarks: A temperate and North European species. It is a psychrophilous species, preferentially inhabiting caves and the runs and nests of small mammals.

*Crumomyia parentela ssp. alpicola (Roháček in Troger \& Roháček, 1980)

Literature: Slovenský kras-Stará brzotínska jaskyňa (cave) (Roháček 1991a), Vlčia jaskyn̆a (cave), Koniarova jaskyňa (cave) (Roháček 1991a; Roháček \& Košel 1993).

Remarks: This psychrophilous subspecies is restricted to the alpine zone of high mountains of Central Europe (Switzerland, Italy, Austria, Slovakia) but can also live in caves at lower montane altitudes (Slovakia). In Slovakia it is a rare species which was, besides the above records from caves, only recorded from Stoh Mt. in the Malá Fatra Mts (Roháček 1983b) and from Alabastrová jaskyňa (cave) in the Belianske Tatry Mts (Roháček \& Košel 2003).

Crumomyia pedestris (Meigen, 1830)

Literature: Slavec nr. Plešivec (Roháček 1986, 1991a).

Remarks: A terricolous, flightless but widespread Palaearctic species distinguished by its wing polymorphism. The strongly brachypterous (micropterous) form usually prevails in populations. It is associated with wetland habitats and its larvae are necrophagous preferentially developing in dead snails (Roháček 1991a).

*Crumomyia pruinosa (Richards, 1932)

Literature: Muránska planina-jaskyňa (cave) Stará Bobačka (Roháček 1991a), Slovenský raj-Vlčia jaskyňa (cave) (Roháček 1991a; Roháček \& Košel 1993).

Material: Muránska planina-Fabova hol'a res., MT, 15.v.-16.vi.2009, 1ठ, J. Ševčík leg. (SMOC).

Remarks: An Arcto-alpine circumpolar species, rarely occurring in the alpine zone of high mountains at more southern latitudes or in caves (in Central Europe: Switzerland, Austria, Czech Republic, Slovakia). In Slovakia, it has hitherto been recorded only from Vel'ká Fatra Mts, Vysoké Tatry Mts. (see Troger \& Roháček 1980) in addition to above records from caves in the Muránska planina Mts and Slovenský raj Mts. The new, non-subterranean finding in the Fabova hol'a Mt. is therefore very important. The species is considered endangered (EN) in the Czech Republic (Roháček 2005).

\section{Crumomyia rohaceki Norrbom \& Kim, 1985}

Literature: Slovenský kras-Hačavská jaskyn̆a (cave), Slovenský raj-jaskyn̆a (cave) Na skale (Roháček 1991a), Slovenský raj-Vlčia jaskyňa (cave), Koniarova jaskyňa (cave) (Roháček 1991a; Roháček \& Košel 1993).

Material: Muránska planina-Fabova hol'a res., MT, 15.v.-16.vi.2009, 1ðึ1, J. Ševčík leg. (SMOC);

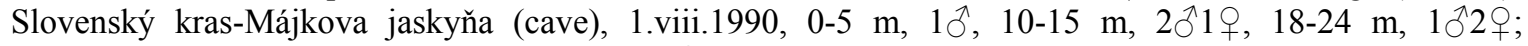
Slovenský kras-Bezodná l'adnica, 4.viii.1990, 1; Slovenský kras-jaskyňa (cave) Milada, 4.viii.1990, 25 m,

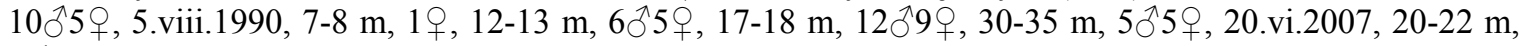
$8{ }^{\lambda} 5$ ㅇ, V. Košel leg. (VKB).

Remarks: A West Palaearctic species, not rare in burrows of small mamals and in caves. Like C. notabilis, adults occur outside cavernicolous habitats only in the cold period of the year. 


\section{*Crumomyia setitibialis (Spuler, 1925)}

Literature: Slovenský raj-Stratenská jaskyňa (cave) (Roháček 1991a), Slovenský raj-Vlčia jaskyňa (cave), Koniarova jaskyňa (cave) (Roháček 1991a; Roháček \& Košel 1993).

Remarks: An Arcto-alpine circumpolar Holarctic species, very rare in alpine zones and caves of Central European montane ranges (only France, Italy, Switzerland, Czech Republic, Slovakia). It inhabits terricolous and subterranean habitats. The above records from Slovenský raj Mts are the only collections known from Slovakia.

*Crumomyia zuskai (Roháček, 1976)

Literature: Slovenský raj-Vlčia jaskyña (cave) (Roháček 1991a; Roháček \& Košel 1993).

Remarks: A rare high montane species (described from the Krkonoše Mts in the Czech Republic and later recorded from mountains in Austria, Slovakia, France, Switzerland but also Spain /Pyrenees Mts/ and Bulgaria /Rila Mts/). The above record (the only from Slovakia) is based on a single male found in the Vlčia jaskyňa (cave) by V. Košel (see Roháček 1991a). The species is considered endangered (EN) in the Czech Republic (Roháček 2005).

Lotophila atra (Meigen, 1830)

Literature: Plešivecká planina, Slavec nr. Plešivec, Brzotín nr. Rožňava, Silická planina, Kečovo, Slovenský kras-Horný vrch, Zádiel, Slovenský raj-sedlo Lipovec (Roháček 1986, 1989).

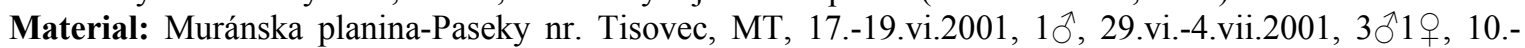

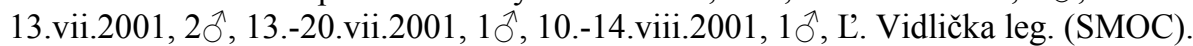

Remarks: A widespread Holarctic, predominantly coprophagous species, very common on dung.

Norrbomia costalis (Zetterstedt, 1847)

Literature: Slovenský raj-sedlo Lipovec (Roháček 1986, 1994a).

Remarks: A West Palaearctic coprophagous species, associated with (preferably horse) dung in pastures.

Norrbomia sordida (Zetterstedt, 1847)

Literature: Slovenský raj-sedlo Lipovec (Roháček 1986, 1994a).

Remarks: A Holarctic coprophagous species, introduced also in Neotropical, Oriental and Oceanian Regions. It occurs on droppings of ungulates (mainly horses) in pastures.

\section{Sphaerocerinae}

\section{Ischiolepta micropyga (Duda, 1938)}

Literature: Slovenský kras-Silická l’adnica, Hačavská dolina-Háj (Roháček 1986).

Remarks: A widespread Palaearctic species but restricted to northern and temperate belts of the region. It is a terricolous species living in damp habitats.

\section{Ischiolepta nitida (Duda, 1920)}

Literature: Slovenský kras-Zádiel (Roháček 1986).

Remarks: A Palaearctic, coprophagous species preferentially occurring in pasture habitats.

Ischiolepta pusilla (Fallén, 1820)

Literature: Muráň-Hrdzavá dolina, Plešivecká planina, Silická planina, Kečovo, Brzotín, Zádielska planina, Turňa nad Bodvou, Slovenský raj-sedlo Lipovec (Roháček 1986).

Remarks: A subcosmopolitan, polysaprophagous (preferably coprophagous), habitat-tolerant and common species.

Ischiolepta vaporariorum (Haliday, 1836)

Literature: Brzotín, Turňa nad Bodvou (Roháček 1986).

Remarks: A widespread Holarctic saprophagous species, rather uncommon in Central Europe.

Lotobia pallidiventris (Meigen, 1830)

Literature: Plešivecká planina, Kečovo (Roháček 1986).

Material: Muránska planina-Paseky nr. Tisovec, MT, 13.-20.vii.2001, 1ð̊, L. Vidlička leg. (SMOC).

Remarks: A markedly thermophilous coprophagous species living in pastures. It is widespread in the Old World but hitherto infrequently encountered in Slovakia.

Sphaerocera curvipes Latreille, 1805

Literature: Muráň-Hrdzavá dolina, Plešivecká planina, Silická l'adnica, Kečovo, Turňa nad Bodvou, Zádiel, Drienovec, Slovenský raj-sedlo Lipovec (Roháček 1986).

Material: Muráň-Hrdzavá dolina, MT, 1.v.-28.vi.2010, 10̂̃, J. Ševčík leg. (SMOC).

Remarks: A very common, cosmopolitan and omnipresent species, abundant in habitats with accumulated dung of large mammals. 


\section{Limosininae}

\section{Apteromyia claviventris (Strobl, 1909)}

Literature: Zádielska dolina (Roháček 1986).

Material: Slovenský kras-priepast' (chasm) Vel'ká Žomboj, 9.v.1981, 1q, V. Košel leg. (VKB).

Remarks: A widespread Holarctic species, known from most of Europe, mainly occurring in leaf litter and various cavernicolous habitats.

*Aptilotus paradoxus Mik, 1898

Literature: Zádielska dolina (Roháček 1986).

Remarks: A Central European apterous species, living in detritus in montane deciduous (mainly beech) forests. It was treated as a lower risk (LR) species in the Slovak red list of Diptera (Jedlička \& Stloukalová 2001).

\section{Bifronsina bifrons (Stenhammar, 1855)}

Literature: Brzotín (Roháček 1986).

Remarks: A subcosmopolitan species associated with various decaying matter. It was formerly classified in the genus Spelobia Spuler, 1924 (subgenus Bifronsina Roháček, 1983).

\section{Chaetopodella scutellaris (Haliday, 1836)}

Literature: Plešivecká planina, Bôrka, Kružná nr. Rožňava, Silická planina, Kečovo, Turňa nad Bodvou, Zádiel, Slovenský raj-sedlo Lipovec, Vel’ký Sokol (Roháček 1983a, 1986), Muráň-Hrdzavá dolina, Plešivec, Brzotín, Drienovec (Roháček 1986).

Material: Muránska planina-Paseky nr. Tisovec, MT, 10.-13.vii.2001, 2ð², L'. Vidlička leg.; Muránska Huta-Šiance res., sweeping undergrowth of beech forest, 7.x.2010, 1q, J. Roháček leg. (SMOC).

Remarks: A widespread Palaearctic, predominantly coprophagous species, most common in pastures.

Coproica acutangula (Zetterstedt, 1847)

Literature: Zádiel, Slovenský raj-sedlo Lipovec (Roháček 1986).

Material: Muránska planina-Paseky nr. Tisovec, MT, 29.vi.-4.vii.2001, 20̂, 10.-13.vii.2001, 10ึ, L'. Vidlička leg. (SMOC).

Remarks: A subcosmopolitan coprophagous species preferentially associated with horse dung in pastures.

\section{Coproica ferruginata (Stenhammar, 1855)}

Literature: Muráň-Hrdzavá dolina, Plešivecká planina, Kečovo, Brzotín, Zádiel, Drienovec, Slovenský rajsedlo Lipovec, Vel'ký Sokol (Roháček 1986).

Material: Muránska planina-Fabova hol’a res., MT, 15.v.-16.vi.2009, 19, 16.vi.-25.viii.2009, 1ठ; MuráňHrdzavá dolina, MT, 1.v.-28.vi.2010, 1ð̄, 28.vi.-26.vii.2010, 1ð̂, all J. Ševčík leg. (SMOC).

Remarks: A very common, cosmopolitan and ubiquitous species, particularly abundant on accumulated dung.

\section{Coproica hirticula Collin, 1956}

Literature: Kružná, Brzotín, Zádiel, Turňa nad Bodvou, Slovenský raj-sedlo Lipovec (Roháček 1986).

Remarks: A common species, subcosmopolitan due to its synanthropy, polysaprophagy and habitat tolerance.

\section{Coproica hirtula (Rondani, 1880)}

Literature: Brzotín, Rožňava, Zádielska planina (Roháček 1986).

Remarks: Another subcosmopolitan synanthropic species, polysaprophagous but less common than $C$. hirticula in Central Europe.

Coproica lugubris (Haliday, 1836)

Literature: Muráň-Hrdzavá dolina, Plešivecká planina, Kružná, Brzotín, Bôrka, Silická planina, Kečovo, Zádielska planina, Turňa nad Bodvou, Slovenský raj-sedlo Lipovec (Roháček 1986).

Remarks: A species widespread in the Oriental and Palaearctic Regions, common on dung in pastures.

Coproica pusio (Zetterstedt, 1847)

Literature: Muráň-Hrdzavá dolina (Roháček 1986).

Remarks: A Palaearctic species also known from Pakistan. It is associated with dung in pastures.

Coproica vagans (Haliday, 1833)

Literature: Muráň-Hrdzavá dolina, Brzotín, Zádiel, Turňa nad Bodvou, Slovenský raj-sedlo Lipovec, Vel'ký Sokol (Roháček 1986).

Material: Muránska planina-Paseky nr. Tisovec, MT, 29.vi.-4.vii.2001, 1ð̂, L. Vidlička leg. (SMOC).

Remarks: A very common, cosmopolitan, polysaprophagous species, mainly living on dung in various habitats. 
Elachisoma aterrimum (Haliday, 1833)

Literature: Plešivecká planina, Kružná, Brzotín, Kečovo, Zádielska planina, Turňa nad Bodvou (Roháček 1986).

Remarks: A Holarctic polysaprophagous species, introduced to some other areas due to its synanthropy.

*Elachisoma bajzae Papp, 1983

Literature: Brzotín nr. Rožňava (Papp 1983, 5つ2ㅇ paratypes), Zádielska planina (Roháček 1986).

Remarks: A Submediterranean thermophilous species, reaching its northern limits in Central Europe. It is considered a vulnerable (VU) species in the Czech Republic (Roháček 2005). Its original description (Papp 1983) was partly based on specimens (paratypes) collected by J. Roháček in the Slovenský kras.

Elachisoma pilosum (Duda, 1924)

Literature: Brzotín (Roháček 1986).

Material: Muráň-Hrdzavá dolina, MT, 1.v.-28.vi.2010, 1ð̋, 28.vi.-26.vii.2010, 1 \& , J. Ševčík leg. (SMOC).

Remarks: A predominantly coprophagous species mainly occurring on pastures. It is widespread in the Palaearctic and Oriental Regions but uncommon in Slovakia,.

Eulimosina ochripes (Meigen, 1830)

Literature: Slovenský raj-Kysel' (Roháček 1983a), Slavec nr. Plešivec, Brzotín, Zádiel (Roháček 1986).

Remarks: A Holarctic phytosaprophagous species, associated with grassland habitats. It was formerly classified within the genus Spelobia Spuler, 1924 (subgenus Eulimosina Roháček, 1983).

Gigalimosina flaviceps (Zetterstedt, 1847)

Material: Muránska planina-Fabova hol’a res., MT, 15.v.-16.vi.2009, 1q, J. Ševčík leg.; Muráň-Hrdzavá dolina, on decayed fungi, 7.x.2010, 1\%; Muránska Huta-Šiance res., sweeping undergrowth of beech forest, 7.x.2010, 2ㅇ, J. Roháček leg. (SMOC); Slovenský kras-jaskyňa (cave) Milada, 8.ix.2010, 5-6 m, 19, 10-12 m, 3ð1오, 20-22 m, 1으. V. Košel leg. (VKB).

Remarks: A distinctive but uncommon European species, occurring in humid forests, on wet leaves between rocks etc., preferably in mountains.

Gonioneura spinipennis (Haliday, 1836)

Literature: Kečovo, Silická planina, Brzotín, Zádiel (Roháček 1983a, 1986), Muráň-Hrdzavá dolina, Kružná, Hačavská dolina-Háj (Roháček 1986).

Material: Muráň-Hrdzavá dolina, MT, 1.v.-28.vi.2010, 1ㅇ, J. Ševčík leg. (SMOC).

Remarks: A common polysaprophagous (mainly coprophagous) species, widespread in the Holarctic Region.

*Herniosina horrida (Roháček, 1978)

Literature: Muráň-Hrdzavá dolina (Roháček 1986), Slovenský kras-Zádielska dolina (Roháček 1978a, as Limosina, 1 male paratype; Roháček 1983a, 1986).

Material: Muránska planina-jaskyňa (cave) Teplica nr. Tisovec, 7.viii.1991, $3{ }^{\lambda} 2$; Muránska planinajaskyňa (cave) Stará Bobačka, 25.viii.1982, 1ð3 $\jmath_{+}$, V. Košel leg. (VKB).

Remarks: A local terricolous and microcavernicolous species known only from Austria, Czech Republic and Slovakia. It was partly described from Slovenský kras (based on male paratype, see Roháček 1978a). It lives in rotten leaf litter or in runs of voles. It is treated as a lower risk (LR) species in the Slovak red list of Diptera (Jedlička \& Stloukalová 2001).

*Herniosina pollex Roháček, 1993

Literature: Slovenský kras-Stará brzotínska jaskyňa (cave) (holotype male, allotype female, paratypes, Roháček 1993), Májkova jaskyňa (cave) nr. Silica (paratypes), Hrabušice-jaskyňa (cave) pod Zelenou horou (paratypes, Roháček 1993).

Remarks: A little known cavernicolous and terricolous species recorded from Germany, Czech Republic, Slovakia and South European Territory of Russia. Besides entrances of caves, it can sometimes be found in ravines and other shaded habitats. Its original description is based on specimens from several Slovak caves including those from Slovenský kras (type locality: Stará brzotínska jaskyňa) and Slovenský raj (see Roháček 1993).

Leptocera caenosa (Rondani, 1880)

Material: Slovenský kras-Jasovská jaskyňa (cave), 2003, sample 657-03, 1ð4오 ? leg. (VKB).

Remarks: A cosmopolitan synanthropic species, living mostly in human settlements, less often in wild on carrion, in burrows of mammals or in caves.

Leptocera fontinalis (Fallén, 1826)

Literature: Muráň-Hrdzavá dolina (Roháček 1986), Slovenský kras-Kečovo, Domica (Roháček 1982, 1986), Slavec, Brzotín, Zádiel, Hačavská dolina-Háj (Roháček 1986).

Material: Muránska planina-Fabova hol'a res., MT, 16.vi.-25.viii.2010, $2{ }^{\Uparrow} 2$ \%, J. Ševčík leg.; Muránska Huta-Šiance res., sweeping undergrowth of beech forest, 7.x.2010, $1 \delta^{\Uparrow} 1$, , J. Roháček leg. (SMOC). 
Remarks: A Holarctic saprophagous species, common both in woodland and open habitats with decayed vegetation.

Leptocera nigra Olivier, 1813

Literature: Jelšavská Teplica (Roháček 1986), Slavec, Plešivec, Slovenský kras-Horný vrch, Zádiel (Roháček 1982, 1986).

Material: Muránska planina-Paseky nr. Tisovec, MT, 29.vi.-4.vii.2001, 2ð1오, L. Vidlička leg.; Muránska Huta-Šiance res., aspirated from tufts of grass, 7.x.2010, 10̄, J. Roháček leg. (SMOC).

Remarks: A widespread Old World phytosaprophagous species, very common in open habitats and warm deciduous forests.

Limosina silvatica (Meigen, 1830)

Literature: Muráň-Hrdzavá dolina (Roháček 1986), Slovenský kras-Kečovo, Zádiel (Roháček 1983a, 1986), Slovenský raj-Vlčie diery cave (Roháček \& Košel 1993).

Material: Muránska planina-Fabova hol'a res., MT, 15.v.-16.vi.2010, 1ðึ, 16.vi.-25.viii.2010, 1ð1, J. Ševčík leg. (SMOC); Muránska planina-jaskyňa (cave) Stará Bobačka, 25.viii.1982, 11ð̃28@; Muránska planina-jaskyňa (cave) Teplica nr. Tisovec, 7.viii.1991, 3 q ; Slovenský kras-Stará brzotínska jaskyňa (cave), 9.vi.1987, 1ठ1; Slovenský kras-Májkova jaskyňa (cave), 1.viii.1990, 0-5 m, 19; Slovenský kras-

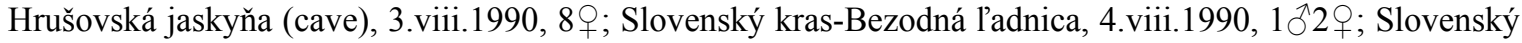
kras-jaskyňa (cave) Milada, 4.viii.1990, 0-5 m, 2, $25 \mathrm{~m}, 6 \jmath^{\Uparrow} 11$, 5.viii.1990, 7-8 m, 1ð6, 12-13 m,

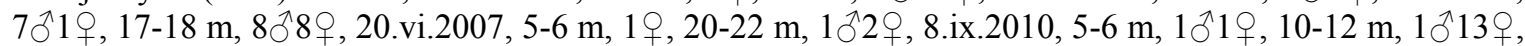
20-22 m, $1 \widehat{\jmath} 4$ 우, V. Košel leg. (VKB).

Remarks: A West Palaearctic phytosaprophagous species, most often occurring on moist rotting leaves in deciduous and mixed forests.

Minilimosina (Minilimosina) fungicola (Haliday, 1836)

Literature: Slovenský kras-Zádiel, Drienovec (Roháček 1986).

Material: Muránska planina-Fabova hol'a res., MT, 16.vi.-25.viii.2010, 1 9 ; Zádielska tiesňava, sweeping, 5.ix.2008, 1 ㅇ, J. Ševčík leg. (SMOC).

Remarks: A common habitat-tolerant, polysaprophagous species, widespread in the Holarctic Region.

Minilimosina (Minilimosina) parvula (Stenhammar, 1855)

Literature: Slovenský kras-Zádielska dolina, Drienovec (Roháček 1986).

Remarks: A common Holarctic polysaprophagous species, with distinct preference for necrophagy and often also occurring on decayed fungi.

Minilimosina (Svarciella) v-atrum (Villeneuve, 1917)

Literature: Slovenský kras-Drienovec, Hačavská dolina-Háj (Roháček 1986, as M. splendens).

A Palaearctic polysaprophagous species restricted to the zone of deciduous forest, not rare in woodland habitats. It was formerly recorded from Slovakia under the synonym M. (S.) splendens (Duda, 1928)

Minilimosina (Svarciella) vitripennis (Zetterstedt, 1847)

Literature: Slovenský raj-Kysel' (Roháček 1983a).

Remarks: A widespread Holarctic species, occurring in various open habitats including meadows and pastures.

Opacifrons coxata (Stenhammar, 1855)

Literature: Vlachovo, Jelšavská Teplica, Slavec, Plešivec, Kečovo, Domica, Brzotín, Hrhov, Slovenský raj-Horný vrch, Zádiel, Turňa nad Bodvou (Roháček 1986).

Material: Muránska planina-Fabova hol’a res., MT, 16.vi.-25.viii.2010, 1q; Muráň-Javorníkova dolina, sweeping, 14.v.2009, 1 + , J. Ševčík leg. (SMOC).

Remarks: A common Palaearctic species living in muddy shores and various marshland habitats.

*Opacifrons moravica (Roháček, 1975)

Literature: Slavec nr. Plešivec (Roháček 1986).

Remarks: A local and rather rare species associated with floodplain or waterlogged forests. Hitherto it was known only from Germany, Czech Republic, Slovakia, Hungary and Bulgaria. The few records from Slovakia are listed by Roháček (1986). The species is considered vunerable (VU) in the Czech Republic (Roháček 2005).

Opalimosina (Hackmanina) czernyi (Duda, 1918)

Literature: Slovenský kras-Drienovec (Roháček 1986).

Remarks: An uncommon Palaearctic mycetophagous species restricted to the zone of deciduous forests.

Opalimosina (Opalimosina) calcarifera (Roháček, 1975)

Literature: Slovenský kras-Hrušov (male holotype), Silická l'adnica (female allotype, see Roháček 1975, as Limosina), these records are also repeated in Roháček (1983a, 1986). 
Remarks: A coprophagous species preferentially living on dung in pastures, known from scattered records throughout the Palaearctic Region. It was originally described from the Slovenský kras (Roháček 1975, type locality: Hrušov).

Opalimosina (Opalimosina) collini (Richards, 1929)

Literature: Muráň-Hrdzavá dolina (Roháček 1986), Slovenský kras-Bôrka, Silická planina (Roháček 1983a, 1986), Brzotín, Zádielska planina, Turňa nad Bodvou (Roháček 1986).

Remarks: A Palaearctic coprophagous species living in pastures and developing in droppings of large mammals.

Opalimosina (Opalimosina) mirabilis (Collin, 1902)

Literature: Muráň-Hrdzavá dolina, Štítnik, Slavec, Brzotín, Zádiel (Roháček 1986), Hrušov, Kružná nr. Rožňava, Turňa nad Bodvou, Slovenský raj-Velký Sokol (Roháček 1983a, 1986).

Material: Muránska planina-Hrdzavá dolina, MT, 1.v.-28.vi.2010, 1ㅇ, 28.vi.-26.vii.2010, 4ð, 26.vii.7.x.2010, 1ð̂1 + , J. Ševčík leg. (SMOC).

Remarks: A subcosmopolitan, polysaprophagous species, mainly associated with dung of domestic animals.

Opalimosina (Opalimosina) simplex (Richards, 1929)

Literature: Muráň-Hrdzavá dolina, Brzotín (Roháček 1986), Slovenský kras-Hrušov (Roháček 1983a, 1986).

Remarks: A European but uncommon coprophagous species occurring on droppings of large mammals.

Opalimosina (Pappiella) liliputana (Rondani, 1880)

Literature: Slovenský kras-Hrušov, Silická l'adnica (Roháček 1983a, 1986).

Remarks: A Holarctic, habitat-tolerant, polysaprophagous species developing in various rotting substrates.

Paralimosina fucata (Rondani, 1880)

Literature: Slovenský kras-Zádielská dolina, Turňa nad Bodvou, Slovenký raj-Vel'ký Sokol (Roháček 1977, as Limosina), Plešivecká planina, Drienovec (Roháček 1986).

Remarks: A West Palaearctic, mainly woodland, species living in forest litter but also attracted to rotting fungi, dung or carrion.

*Paralimosina kaszabi Papp, 1973

Material: Muránska planina-Fabova hol’a res., MT, 16.vi.-25.viii.2010, 1, J. Ševčík leg.; Muránska planina-Paseky nr. Tisovec, MT, 17.-19.vi.2001, 2ᄋ, 29.vi.-4.vii.2001, 2ð̂, 4.-6.vii.2001, 1ठ,, L'. Vidlička leg. (SMOC).

Remarks: A Palaearctic, rare terricolous and microcavernicolous species, occurring in wet open habitats.

Paralimosina trichopyga (Richards, 1952)

Material: Muránska planina-Fabova hol’a res., MT, 15.v.-16.vi.2010, 19, 16.vi.-25.viii.2010, 8ð3q, J. Ševčík leg. (SMOC).

Remarks: A terricolous woodland species known only from Central Europe (Austria, Czech Republic, France, Germany, Slovakia, Switzerland). It occurs infrequently in montane forests.

Philocoprella italica (Deeming, 1964)

Literature: Kružná, Brzotín, Slovenský kras-Silická planina, Turňa nad Bodvou (Roháček 1986).

Remarks: A Palaearctic thermophilous coprophagous species, encountered infrequently on dung in pastures.

Phthitia (Kimosina) plumosula (Rondani, 1880)

Literature: Muráň-Hrdzavá dolina, Slavec nr. Plešivec, Hačavská dolina-Háj (Roháček 1986, as Kimosina). Remarks: A phytosaprophagous species widespread in the Holarctic Region but also recorded from tropics. It preferentially occurs in deciduous forests.

Pseudocollinella humida (Haliday, 1836)

Literature: Vlachovo, Jelšavská Teplica, Štítnik, Slavec nr. Plešivec, Brzotín, Silica, Domica, Kečovo, Horný vrch, Zádielske Dvorníky, Drienovec (Roháček 1986, as Opacifrons).

Material: Muránska planina-Hrdzavá dolina, MT, 28.vi.-26.vii.2010, 6ð4@, 26.vii.-7.x.2010, $2 \jmath^{\jmath}$, J. Ševčík leg. (SMOC).

Remarks: A very common Palaearctic paludicolous species living in various muddy habitats.

*Pseudocollinella jorlii (Carles-Tolrá, 1990)

Literature: Slavec nr. Plešivec (Roháček 2001).

Remarks: A mainly Mediterranean and Macaronesian species rarely occurring in Great Britain, in Central Europe only recorded from two localities in Slovakia (Roháček 2001: Slavec nr. Plešivec and Bot’any nr. Latorica river). It is a mud-loving species occurring on banks of rivers. 


\section{Pteremis fenestralis (Fallén, 1820)}

Material: Muránska Huta-Šiance res., aspirated from tufts of grass, 7.x.2010, 10ิ, J. Roháček leg. (SMOC). Remarks: A Palaearctic, terricolous species inhabiting grass and moss litter in wooded as well as open habitats. It is a wing-polymorphic species but only the macropterous form was found in the study area.

Pullimosina (Pullimosina) heteroneura (Haliday, 1836)

Literature: Slavec nr. Plešivec (Roháček 1986).

Remarks: A common cosmopolitan, polysaprophagous species, living in various rotting substrates.

Pullimosina (Pullimosina) meijerei (Duda, 1918)

Literature: Slovenský kras-Zádielska dolina (Roháček 1978b, as Limosina, Roháček 1986).

Remarks: A wing-polymorphic species living in moss, leaf litter and rotten grass in woodland as well as open habitats. It seems to be restricted to North and temperate Europe.

Pullimosina (Pullimosina) moesta (Villeneuve, 1918)

Literature: Muráň-Hrdzavá dolina, Slovenský kras-Drienovec (Roháček 1986, as $P$. antennata).

Remarks: A West Palaearctic phytosaprophagous species, but less frequent than the related P. vulgesta. It preferentially occurs in open habitats and was formerly recorded under the synonymic name $P$. antennata (Duda, 1918).

Pullimosina (Pullimosina) pullula (Zetterstedt, 1847)

Literature: Plešivec, Slavec, Zádiel, Drienovec (Roháček 1986).

Remarks: A Holarctic terricolous species, living in plant debris in meadows and forests. It is parthenogenetic in Central Europe.

Pullimosina (Pullimosina) vulgesta Roháček, 2001

Literature: Slovenský kras-Hrušov (Roháček 1878b, as Limosina moesta), Plešivec, Slavec, Zádiel, Hačavská dolina-Háj, Drienovec (Roháček 1986, as Pullimosina moesta).

Remarks: A West Palaearctic species, very common in grassland habitats in Central Europe. It was formerly misrecorded as Limosina or Pullimosina moesta.

Puncticorpus cribratum (Villeneuve, 1917)

Literature: Slovenský kras-Drienovec (Roháček \& Marshall 1982; Roháček 1986).

Remarks: A West Palaearctic terricolous species living in leaf litter under rotting sporocarps of fungi in which its larvae develop. The species is predominantly brachypterous in Central and North Europe but macropterous at more southern latitudes.

* Rachispoda cilifera (Rondani, 1880)

Literature: Slavec nr. Plešivec (Roháček 1991b, as Leptocera (Rachispoda)).

Remarks: An uncommon thermophilous species distributed in lowlands of Central and South Europe. It preferentially occurs on mud in floodplain forests at large rivers (Roháček 1991b) and is considered vunerable (VU) in the Czech Republic (Roháček 2005).

Rachispoda hostica (Villeneuve, 1917)

Literature: Silica, Horný vrch-Krasové jazierko (Roháček 1986, 1991b, both Leptocera (Rachispoda)).

Remarks: Another uncommon but widespread Palaearctic species associated with marshland and muddy habitats. It was infrequently encountered in Slovakia (cf. Roháček 1991b).

Rachispoda limosa (Fallén, 1820)

Literature: Jelšavská Teplica, Slavec, Plešivec, Brzotín, Silica, Domica, Horný Vrch, Zádielske Dvorníky (Roháček 1986, 1991b, both as Leptocera (Rachispoda)).

Remarks: A common Holarctic paludicolous species in muddy habitats along waters and in marshlands.

Rachispoda lutosa (Stenhammar, 1855)

Literature: Vlachovo, Jelšavská Teplica, Slavec, Plešivec, Brzotín, Silica, Domica, Horný Vrch, Hrhov, Turňa nad Bodvou, Hačavská dolina-Háj (Roháček 1986, 1991b, both as Leptocera (Rachispoda)).

Remarks: A very common paludicolous Holarctic species living on shores of water bodies, in marshy meadows and forests.

Rachispoda lutosoidea (Duda, 1938)

Literature: Vlachovo, Jelšavská Teplica, Slavec, Plešivec, Brzotín, Gombasek, Silica, Domica, Horný Vrch-Krasové jazierko, Hrhov, Turňa nad Bodvou, Zádielske Dvorníky (Roháček 1986, 1991b, both as Leptocera (Rachispoda)).

Remarks: A West Palaearctic species occuring on mud on shores of water bodies, in boggy meadows and forests.

Rachispoda modesta (Duda, 1924)

Literature: Muráň-Hrdzavá dolina, Jelšavská Teplica, Slavec, Plešivec, Brzotín, Horný Vrch-Krasové jazierko, Hrhov, Zádielske Dvorníky (Roháček 1986, 1991b, both as Leptocera (Rachispoda)). 
Material: Muráń-Javorníkova dolina, sweeping, 14.v.2019, 10ึ, J. Ševčík leg. (SMOC).

Remarks: A West Palaearctic species, living in muddy habitats, mostly in lower altitude wetlands.

*Rachispoda pseudohostica (Duda, 1924)

Literature: Jelšavská Teplica, Turňa nad Bodvou (Roháček 1983b, 1986, 1991b, all as Leptocera (Rachispoda)).

Remarks: A Palaearctic species, thermophilous and hence uncommon in C. Europe, occurring on mud along water bodies in lowland areas. Its first records from Slovakia originate from the above localities (Roháček 1983b). The species is considered endangered (EN) in the Czech Republic (Roháček 2005).

*Rachispoda segem (Roháček, 1991)

Literature: Slavec nr. Plešivec (Roháček 1991b, as Leptocera (Rachispoda), $1{ }^{\Uparrow} 1$ 1 paratypes).

Remarks: A little known European species associated with natural shady river banks and occurring on mud or in riparian vegetation (Roháček 1991b). It was partly described from specimens collected by the author in the above locality. The species is considered vulnerable (VU) in the Czech Republic (Roháček 2005).

*Spelobia cambrica (Richards, 1929)

Literature: Muráň-Hrdzavá dolina, Zádielska dolina, Drienovec (Roháček 1986), Slovenský raj-Velký Sokol (Roháček 1983a).

Material: Muráň-Hrdzavá dolina, MT, 1.v.-28.vi.2010, 19, 26.vii.-7.x.2010, 19, J. Ševčík leg., same locality, on decayed fungi, 7.x.2010, 9 3 , J. Roháček leg.; Muránska Huta-Šiance res., sweeping undergrowth of beech forest, 7.x.2010, 10̂, J. Roháček leg. (SMOC).

Remarks: A Palaearctic polysaprophagous species associated with well-preserved montane forests and also peat bogs.

Spelobia clunipes (Meigen, 1830)

Literature: Muráň-Hrdzavá dolina, Slavec, Brzotín, Turňa nad Bodvou, Drienovec, Hačavská dolina-Háj (Roháček 1986), Silická l’adnica, Kečovo, Zádielska dolina, Slovenský raj-sedlo Lipovec, Vel'ký Sokol (Roháček 1983a, 1986).

Material: Muránska planina-Paseky nr. Tisovec, MT, 10.-13.vii.2001, 1§1우, L. Vidlička leg.; Muránska

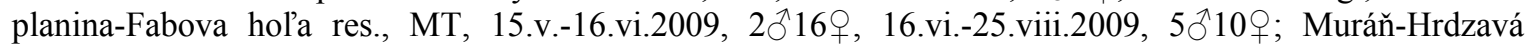
dolina, MT, 26.vii.-7.x.2010, 10े; Muráň-Javorníkova dolina, sweeping, 14.v.2009, 3 q , all J. Ševčík leg.; Muránska Huta-Šiance res., sweeping undergrowth of beech forest, 7.x.2010, 201우, J. Roháček leg. (SMOC); Slovenský kras-priepast' (chasm) Vel'ká Žomboj, 9.v.1981, 19; Slovenský kras-Stará brzotínska jaskyňa (cave), 9.vi.1987, 10ิ; Slovenský kras-Májkova jaskyňa (cave), 1.viii.1990, 0-5 m, 10̂; Slovenský kras-jaskyňa (cave) Milada, 4.viii.1990, 0-5 m, $5{ }^{\lambda} 8$ \% ; Slovenský raj-Medvedia jaskyňa (cave), 30.vii.1989, 10-15 m, $1{ }^{\Uparrow} 1$ 우, V. Košel leg. (VKB).

Remarks: A Holarctic polysaprophagous and very common species developing in various rotting substrates.

Spelobia czizeki (Duda, 1830)

Literature: Jelšavské Teplice (Roháček 1986), Kečovo-Kečovská vyvieračka cave (Roháček 1983a).

Material: Slovenský kras-Hrušovská jaskyňa (cave), 3.viii.1990, 10̂; Slovenský kras-Májkova jaskyňa (cave), 1.viii.1990, 0-5 m, 2q; Slovenský kras-jaskyňa (cave) Milada, 4.viii.1990, 0-5 m, 10̂, 10-35 m, 10

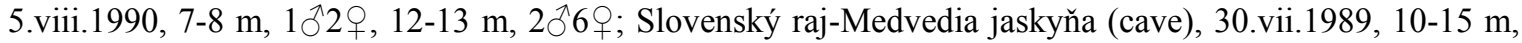
$3 \bigcirc^{\lambda} 12$ ㅇ, 20-25 m, 1ㅇ,V. Košel leg. (VKB).

Remarks: An uncommon Palaearctic species occuring in various subterranean habitats (caves, cellars, burrows of mammals).

*Spelobia faeroensis (Deeming, 1966)

Literature: Plešivecká planina, Kečovo (Roháček 1983a, 1986).

Remarks: A very rare species known only from limited records from Faeroe I. (Denmark), Switzerland and from above two localities in Slovakia. It is probably saprophagous as is its closest relative S. clunipes.

Spelobia ibrida Roháček, 1983

Literature: Muráň-Hrdzavá dolina (Roháček 1986).

Material: Muránska planina-Fabova hol’a res., MT, 16.vi.-25.viii.2009, 10 , J. Ševčík leg. (SMOC).

Remarks: A relatively rare European phytosaprophagous species. It seems to be restricted to montane forests in Central and South Europe.

Spelobia luteilabris (Rondani, 1880)

Literature: Turňa nad Bodvou (Roháček 1986).

Remarks: A Holarctic species, polysaprophagous and living in various habitats from lowlands to mountains. 
Spelobia manicata (Richards, 1927)

Material: Muránska planina-Fabova hol’a res., MT, 16.vi.-25.viii.2009, 1ð̂; Muráň-Hrdzavá dolina, MT,

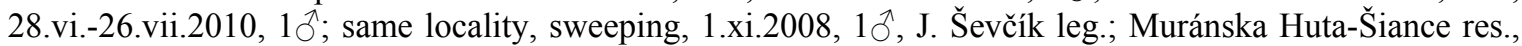
sweeping undergrowth of beech forest, 7.x.2010, 10, J. Roháček leg. (SMOC).

Remarks: A European microcavernicolous species developing in rotting matter in runs and nests of small mammals.

Spelobia nana (Rondani, 1880)

Material: Muránska Huta-Šiance res., aspirated from tufts of grass, 7.x.2010, 1ðð, J. Roháček leg. (SMOC). Remarks: A European species found also in the Nearctic Region (possibly introduced). It is uncommon in

C. Europe occurring in open grassland habitats preferentially developing in buried carrion of small animals.

Spelobia palmata (Richards, 1927)

Literature: Muráň-Hrdzavá dolina, Zádielska dolina (Roháček 1986), Kečovo (Roháček 1983a).

Material: Muránska planina-Fabova hol'a res., MT, 15.v.-16.vi.2009, 1ㅇ, 16.vi.-25.viii.2009, $2 \precsim 2$ 잉. J. Ševčík leg.; Muráň-Hrdzavá dolina, sweeping undergrowth of beech forest, 7.x.2010, 1우, J. Roháček leg. (SMOC).

Remarks: A West Palaearctic, mainly necrophagous species, common in woodland on carrion and decayed fungi.

Spelobia parapusio (Dahl, 1909)

Literature: Muráň-Hrdzavá dolina, Zádielska dolina, Drienovec (Roháček 1986).

Material: Muránska planina-Fabova hol'a res., MT, 15.v.-16.vi.2009, 19, 16.vi.-25.viii.2009, 19; Murán̆Hrdzavá dolina, MT, 1.v.-28.vi.2010, 1ㅇ, 28.vi.-26.vii.2010, 1ㅇ, 26.vii.-7.x.2010, 2ㅇ, all J. Ševčík leg.; same locality, on decayed fungi, 7.x.2010, 8 8 ; Muránska Huta-Šiance res., sweeping undergrowth of beech forest, 7.x.2010, 1우 J. Roháček leg.; Zádielska tiesňava, sweeping, 5.ix.2008, 1웅 Drienovec, sweeping, 5.ix.2008, 1ㅇ, J. Ševčík leg. (SMOC).

Remarks: A Palaearctic mycetophagous species, parthenogenetic in Central Europe, common in forested habitats on decaying fungi.

Spelobia pseudosetaria (Duda, 1918)

Literature: Kečovo (Roháček 1983a, 1986).

Remarks: Holarctic species also introduced into New Zealand due to its synanthropy. For unknown reasons it seems to have become recently uncommon in C. Europe despite its polysaprophagy and habitat tolerance.

Spelobia rufilabris (Stenhammar, 1855)

Literature: Hačavská dolina-Háj (Roháček 1986).

Remarks: A widespread Palaearctic species, occurring rather frequently in various decayed vegetable debris in montane forests or mires.

*Spelobia simplicipes (Duda, 1925)

Literature: Plešivecká planina, Kečovo (Roháček 1983a, 1986).

Remarks: An uncommon coprophagous species distributed in southern Palaearctic and reaching its northernmost limits in Central Europe. Because of its thermophily it is very rare in Slovakia (only 5 specimens were found in the two localities above, see Roháček 1983a).

*Spelobia talis Roháček, 1983

Material: Muránska planina-Paseky nr. Tisovec, MT, 4.-6.vii.2001, 1ㅇ, L. Vidlička leg. (SMOC).

Remarks: A scarce and poorly known microcavernicolous species living in runs and nests of small mammals recorded from Spain, Switzerland, Germany, Czech Republic and Slovakia (Roháček et al. 2001). In Slovakia it was previously found in only two localities, viz. Chočské vrchy Mts-Štútova dolina valley and Ruský Potok (Roháček 1994b, 1995).

Spelobia talparum (Richards, 1927)

Literature: Štítnik, Hačavská dolina-Háj (Roháček 1986).

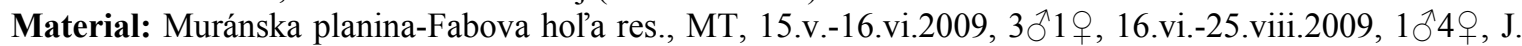

Ševčík leg.; Muránska planina-Paseky nr. Tisovec, MT, 4.-6.vii.2001, 1ㅇ, L. Vidlička leg. (SMOC).

Remarks: A common Palaearctic species inhabiting subterraneous nests and runs of various mammals.

Telomerina flavipes (Meigen, 1830)

Literature: Rožňava (Roháček 1983a, 1986).

Remarks: A subcosmopolitan species because of partial synanthropy, predominantly necrophagous.

Telomerina pseudoleucoptera (Duda, 1924)

Literature: Muráň-Hrdzavá dolina (Roháček 1986), Brzotín (Marshall \& Roháček 1984; Roháček 1986).

Remarks: A European species occurring on dung of large mammals, preferably in pastures. 


\section{Terrilimosina racovitzai (Bezzi, 1911)}

Literature: Silická planina-Ardovská jaskyňa (cave), Silická planina-pripast' (chasm) Brázda (Roháček 1994b).

Material: Slovenský kras-Drienovská jaskyňa, 4.iv.-14.x.2008, bat's hall, ST, 63̊6우, L. Kováč leg., same locality 26.iii.2009, 50 m, on bat's guano, 2 , , 2009, sample 906-09, 30े, A. Mock leg.; Slovenský kras-

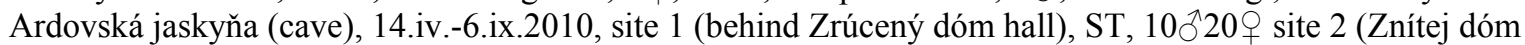
hall), ST, $8{ }^{3} 4$ 우, site 3 (main passage nr. crossing), ST, 1933 우, site 3 (side passage in front of guard), ST, $6{ }^{\lambda} 8$, site 5 (bottom of entrance shaft), ST, $263^{\lambda} 8$, all A. Mock leg.; Slovenský kras-jaskyňa (cave)

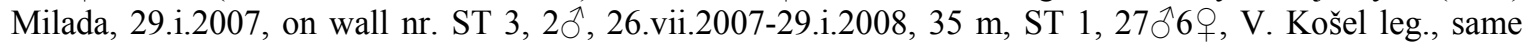
locality, 15.iv.-18.ix.2010, site 5, ST, 27 9 9 , A. Mock \& L'. Kováč leg. (all VKB).

Remarks: A common Holarctic cave-dwelling species classified as hemitroglophilous. Besides caves it can also be found in cellars and (more rarely) in burrows of small mammals. In Slovakia, it has hitherto been recorded only from several caves (Roháček 1994b). However, it proved to be a common species in some cave systems in the Gemer area (see material examined).

Terrilimosina schmitzi (Duda, 1918)

Literature: Muráń-Hrdzavá dolina, Slavec (Roháček 1986), Kečovo, Zádiel (Roháček 1983a, 1986).

Material: Muránska planina-Fabova hol’a res., MT, 16.vi.-25.viii.2009, 4 ${ }^{3} 3$ 우 ; Muráň-Hrdzavá dolina, MT, 28.vi.-26.vii.2010, $5 \bigcirc 2$, 26.vii.-7.x.2010, $1{ }^{\lambda} 2$; same locality, sweeping, 1.xi.2008, 1 ; , all J. Ševčík leg. (SMOC).

Remarks: A Holarctic species, developing in forest litter and common in various woodland habitats.

Trachyopella (Nudopella) leucoptera (Haliday, 1836)

Literature: Brzotín (Roháček \& Marshall 1986; Roháček 1986).

Remarks: A widespread Holarctic saprophagous species also introduced to Hawaii. It can be encountered on dung as well as other decaying substrates in open habitats.

Trachyopella (Trachyopella) atomus (Rondani, 1880)

Literature: Brzotín (Roháček \& Marshall 1986; Roháček 1986).

Remarks: A coprophagous-saprophagous Palaearctic species, also recorded from the Oceanian Region (Guam, Hawaii). Recently, it has become rare in Europe due to competition with the introduced and more successful T. lineafrons. There are only two records of this species from Slovakia, the above from Brzotín and another from Vel'ká Fatra-Suchá dolina (Roháček \& Marshall 1986).

Trachyopella (Trachyopella) kuntzei (Duda, 1918)

Literature: Turňa nad Bodvou (Roháček \& Marshall 1986; Roháček 1986).

Remarks: A West Palaearctic species, also found in Canada (probably introduced). It lives in rotting debris.

Trachyopella (Trachyopella) lineafrons (Spuler, 1925)

Literature: Brzotín, Hrušov, Turňa nad Bodvou (Roháček \& Marshall 1986; Roháček 1986).

Remarks: A subcosmopolitan (originally probably Nearctic) species due to synanthropy, polysaprophagous, developing in various rotting substrates.

Trachyopella (Trachyopella) melania (Haliday, 1836)

Literature: Kružná, Brzotín (Roháček \& Marshall 1986; Roháček 1986).

Remarks: A Palaearctic species, uncommon in Slovakia. It occurs on dung, refuse heaps, decayed hay etc.

Trachyopella (Trachyopella) straminea Roháček \& Marshall, 1986

Literature: Brzotín (Roháček \& Marshall 1986, $3{ }^{\hat{2}} 6$ 우 paratypes).

Remarks: An originally Nearctic species introduced to Europe. Its description (Roháček \& Marshall 1986) was based on specimens both from North America and Europe (and partly /paratypes/ also from the above locality in Slovenský kras). It is largely a phytosaprophagous species occurring in decayed stray, hay, mown grass and similar substrates.

\section{Discussion and conclusions}

A total of 106 species recorded from the Gemer area demonstrates the remarkably high diversity of Sphaeroceridae in this territory which is almost the same as in two other most intensively studied regions of Slovakia, viz. Bukovské vrchy Mts (Roháček 1995 - 101 species) and Pol'ana Mts (Roháček 2009b - 106 species). However, not only the high species diversity but particularly the qualitative composition of the regional sphaerocerid fauna characterize this naturally exceptional area. There is a remarkable mixture of faunal elements in the Gemer terriotory including Arcto-alpine and Boreo-montane or high-montane species in the highest altitudes and in cave systems, typical silvicolous species in well-preserved 

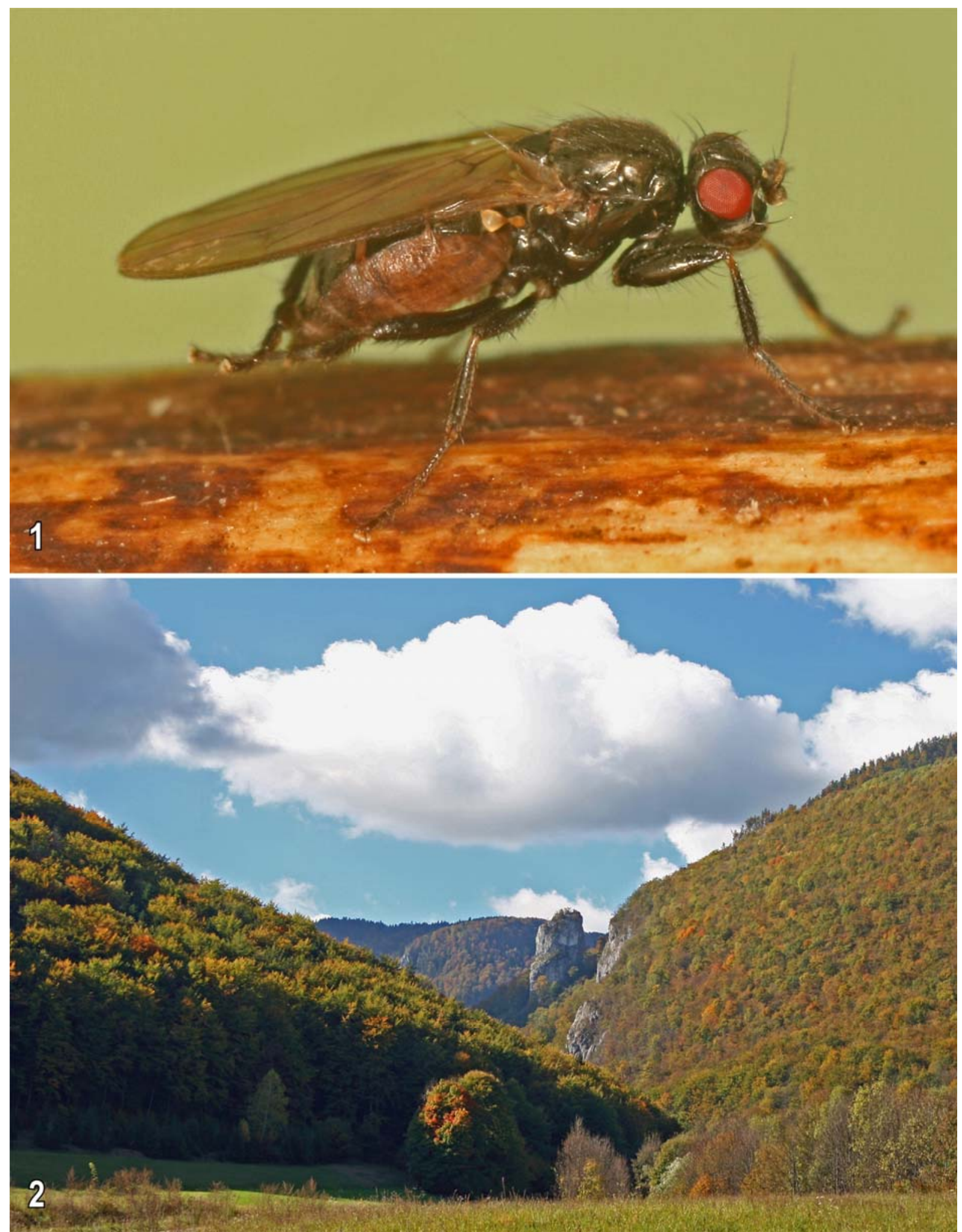

Figs 1-2: 1 - Crumomyia fimetaria (Meigen, 1830), a mycophagous species common in woodland habitats (female from Hrdzavá dolina nr Muráň); 2 - Hrdzavá dolina (viewed from Muráň), a locality with rich communities of Diptera including Sphaeroceridae. 
beech forests, markedly thermophilous coprophagous species of southern origin in foreststeppe and steppe habitats but also paludicolous species restricted to natural muddy shores of rivers in lowlands. A number of them belong to rare, local and/or endangered taxa.

First, it should be stressed that five species of Sphaeroceridae were described (at least partly) from the area.Two of them have their type locality here (i.e. their holotypes were collected in Gemer), viz. Herniosina pollex and Opalimosina (Opalimosina) calcarifera and descriptions of the remaining three species (Elachisoma bajzae, Herniosina horrida, Rachispoda segem) were mostly based on paratypes originating from this territory. In addition, four rare species have hitherto been recorded in Slovakia only from the Gemer area, viz. Crumomyia setitibialis, C. zuskai, Spelobia faeroensis and Spelobia simplicipes.

Ecologically significant also is the occurrence of Arcto-alpine, Boreo-montane and/or high-montane species Copromyza montana, Crumomyia parentela alpicola, C. pruinosa, $C$. setitibialis, C. zuskai. They were found only at the highest altitudes of the Muránska planina Mts (Copromyza montana, Crumomyia pruinosa) or are restricted to cold aphotic habitats in caves (all above species of Crumomyia), see also below.

The Gemer territory also hosts several infrequently encountered silvicolous species characteristic of well-preserved forest habitats (beech forests in particular): Aptilotus paradoxus, Gigalimosina flaviceps, Minilimosina (Svarciella) v-atrum, Opalimosina (Hackmanina) czernyi, Paralimosina trichopyga, Puncticorpus cribratum, Spelobia ibrida, S. cambrica. Another interesting faunal element is composed of thermophilous coprophagous species associated with warm, steppe-like pasture habitats: Lotobia pallidiventris, Elachisoma bajzae, Philocoprella italica, Trachyopella (Trachyopella) straminea and, particularly, Spelobia simplicipes which is not known from other parts of Slovakia. There are also thermophilous paludicolous species in this territory which only occur on natural muddy or sandy banks of lower altitude rivers both in open habitats and floodplain forests: Opacifrons moravica, Pseudocollinella jorlii, Rachispoda cilifera, R. pseudohostica, R. segem.

Cave-dwelling Sphaeroceridae. The Gemer is an area with well-developed carst phenomena including chasms and rich cave systems. The caves represent a special environment inhabited by several species of Sphaeroceridae. Following the classification by Roháček \& Papp (2000), no troglobiont species of Sphaeroceridae occur in the Gemer area (nor in Slovakia as a whole). Of the 18 sphaerocerid species found in Gemer caves only two (Crumomyia parentela alpicola, C. setitibialis), which can spend their entire life-history in deep aphotic zones of the caves, can be classified as troglophilous. The majority of species recorded from these caves are hemitroglophilous. This group includes various psychrophilous species which are, however, not strictly associated with caves, e.g. the high montane or Boreo-alpine species (Crumomyia zuskai, C. pruinosa), various subterranean species living also in runs and burrows of mammals (Crumomyia notabilis, C. rohaceki, Apteromyia claviventris, Herniosina horrida, H. pollex, Spelobia czizeki, Terrilimosina racovitzai), woodland dark-loving species preferentially occurring near entrances of caves (Crumomyia nitida, Gigalimosina flaviceps, Limosina silvatica) but, interestingly, also the coprophagous Crumomyia nigra usually developing in dung on pastures, the predominantly necrophagous and synanthropic Leptocera caenosa or the ubiquitous, extremely habitat-tolerant and polysaprophagous Spelobia clunipes. All these species occur in caves because they are cold and dark tolerant and can utilise decaying organic matter in this habitat as breeding substrate for their larvae (bat guano and rotting vegetable debris in particular). The last species found in the caves of Gemer is the trogloxenous Crumomyia fimetaria. It surely occurs in caves by chance as it is a mycophagous species normally living in woodland.

Endangered Sphaeroceridae. Several species important from a nature-conservancy point of view also live in the Gemer area. Those treated as endangered or vulnerable in the red list of the Czech Republic (Roháček 2005) should be particularly mentioned: Crumomyia 
pruinosa, C. zuskai, Rachispoda pseudohostica (all EN), Elachisoma bajzae, Opacifrons moravica, Rachispoda cilifera, R. segem (all VU). Additional species occurring in the Gemer area [not listed by Roháček (2005) because hitherto not recorded from the Czech Republic] should also be considered as jeopardized in Slovakia (and the whole of Central Europe), apparently in category EN, viz. Crumomyia parentela alpicola, C. setitibialis, Pseudocollinella jorlii, Spelobia faeroensis, S. simplicipes. These rare and very local taxa particularly demonstrate the high natural merit of the Gemer territory.

Acknowledgements: A part of the material presented in this study was collected by J. Ševčík (Silesian Museum, Opava) within the framework of the All Taxa Biodiversity Inventories project (ATBI, see www.atbi.eu) financed by the European Union. I should like to acknowledge him for allowing me to study this material. The kind assistance of the staff (especially K. Necpálová) of the Administration of the Muránska planina National Park is also much appreciated. K. N. Barber (Sault Ste. Marie, Ontario, Canada) is sincerely thanked for comments on the manuscript of this paper. I am particularly grateful to V. Košel (Bratislava, Slovakia) who sent me samples of Sphaeroceridae for examination that were collected by him and his collaborators in caves of the Gemera area and to L. Vidlička (Bratislava, Slovakia) for samples from his Malaise trap. The author's research on Diptera in the Gemer area was supported by a grant from the Silesian Museum no. IGS201103.

\section{References}

Fritsch K. (1875): Jährliche Periode der Insektenfauna von Österreich-Ungarn. I. Die Fliegen (Diptera). Denkschr. k.eiser. Akad. Wiss., Wien 34(1): 33-114. Also published separately with the same title: 82 pp., In Commission bei Karl Gerold's Sohn, Buchändler der kaiserlichen Akademie der Wissenschaften, Wien.

Jedlička L. \& Stloukalová V. (2001): Červený (ekosozologický) zoznam dvojkrídlovcov (Diptera) Slovenska. Red (ecosozological) list of Diptera of Slovakia, pp. 139-142. In Baláž D., Marhold K. \& Urban P. (eds): Červený zoznam rastlín a živočichov Slovenska. Red list of plants and animals of Slovakia. Ochrana Prírody 20 (Suppl.), Bratislava, 160 pp.

Marshall S. A. \& Roháček J. (1984): A revision of the genus Telomerina Roháček (Diptera, Sphaeroceridae). - Syst. Entomol. 9: 127-163.

Papp L. (1983): Three new species of Elachisoma Rondani, 1880 (Diptera: Sphaeroceridae). - Acta Zool. Acad. Sci. Hung. 29: 451-459.

Roháček J. (1975): Three new species of the genus Limosina Macq. (Sphaeroceridae, Diptera) from Czechoslovakia. - Scripta Fac. Sci. Nat. Univ. Purk. Brun., Biologia 2, 5: 115-126.

- (1977): Revision of the Limosina fucata species-group, with descriptions of four new species (Diptera, Sphaeroceridae). - Acta Entomol. Bohemoslov. 74: 398-418.

- (1978a): Limosina horrida sp.n., a new species from Czechoslovakia related to Limosina bequaerti (Vill.) (Diptera, Sphaeroceridae). - Čas. Slez. Muz. Opava (A), 27: 49-60.

- (1978b): Revision of the European species of the Limosina heteroneura-group (Diptera, Sphaeroceridae). Čas. Slez. Muz. Opava (A), 27: 125-151.

- (1982): Revision of the subgenus Leptocera (s. str.) of Europe (Diptera, Sphaeroceridae). - Entomol. Abh. Staat. Mus. Tierk. Dresden 46: 1-44.

- (1983a): A monograph and re-classification of the previous genus Limosina Macquart (Diptera, Sphaeroceridae) of Europe. Part II. - Beitr. Entomol. Berlin 33: 3-195.

- (1983b): Nové nálezy druhů čeledi Sphaeroceridae (Diptera) v Československu. [New records of Sphaeroceridae (Diptera) from Czechoslovakia]. - Čas. Slez. Muz. Opava (A), 32: 85-87.

- (1986): Čel'ad' Sphaeroceridae, pp. 149-164. In Čepelák, J. (ed.): Diptera Slovenska II (Cyclorrhapha). [Diptera of Slovakia II (Cyclorrhapha)], Veda, Bratislava, 435 pp.

- (1989): Sphaeroceridae (Diptera) of Czechoslovakia. Part 1. Lotophila Lioy and Copromyza Fallén. - C̆as. Slez. Muz. Opava (A), 38: 1-16.

- (1991a): Sphaeroceridae (Diptera) of Czechoslovakia. Part 2. Crumomyia Macquart. - Čas. Slez. Muz. Opava (A), 40: 1-27.

- (1991b): A monograph of Leptocera (Rachispoda Lioy) of the West Palaearctic area (Diptera, Sphaeroceridae). - Čas. Slez. Muz. Opava (A), 40: 97-288.

- (1992): Sphaeroceridae (Diptera) of Czechoslovakia. Part 3. Alloborborus Duda and Copromyza montana sp.n. - Čas. Slez. Muz. Opava (A), 41: 193-203.

- (1993): Herniosina Roháček and Minilimosina Roháček of Europe: two new species, new records and taxonomic notes (Insecta, Diptera: Sphaeroceridae). - Entomol. Abh. Staat. Mus. Tierk. Dresden 55(12): $185-203$ 
- (1994a): Sphaeroceridae (Diptera) of Czechoslovakia. Part 4. Norrbomia Papp and Borborillus Duda. - Čas. Slez. Muz. Opava (A), 43: 1-14.

- (1994b): Sphaeroceridae (Diptera) of Slovakia: corrections and additions to faunal list. - Entomol. Problems 25(1): 83-91.

- (1995): Sphaeroceridae, pp. 163-171. In Roháček J., Starý J., Martinovský J. \& Vála M. (eds): Diptera Bukovských vrchov (Diptera of the Bukovské Hills). SAŽP - Správa CHKO a BR Východné Karpaty, Humenné (in Slovak, with English summary), 232 pp.

- (2001): Faunistic records: Sphaeroceridae. In: Dipterologica bohemoslovaca Vol. 10. - Acta Universitatis Carolinae, Biologica 45(1-2): 198.

- (2005): Sphaeroceridae (mrvnatkovití), pp. 344-346. In Farkač J., Král D. \& Škorpík M. (eds): Červený seznam ohrožených druhů České republiky. Bezobratlí. Red list of threatened species in the Czech Republic. Invertebrates. Agentura ochrany př́rody a krajiny České republiky, Praha, 760 pp.

- (2009a): Sphaeroceridae Macquart, 1835. In Jedlička L., Kúdela M. \& Stloukalová V. (eds): Checklist of Diptera of the Czech Republic and Slovakia. Electronic version 2. $<$ http://zoology.fns.uniba.sk/diptera2009> + CD-ROM: ISBN 978-80-969629-4-5.

- (2009b): Sphaeroceridae, pp. 260-271. In Roháček J. \& Ševčík J. (eds): Diptera of the Pol'ana Protected Landscape Area - Biosphere Reserve (Central Slovakia), SNC SR, Administration of the PLA - BR Pol'ana, Zvolen, $340 \mathrm{pp}$

Roháček J. \& Košel V. (1993): Temporal and spacial distribution of Sphaeroceridae (Diptera) in two caves in the Western Carpathians (Slovakia). - Čas. Slez. Muz. Opava (A), 42: 235-257.

- (2003): Crumomyia glacialis (Meigen, 1830) in Slovakia, with description of the female and notes on variation and biology (Diptera, Sphaeroceridae). - Čas. Slez. Muz. Opava (A), 51(2002): 193-198.

Roháček J. \& Marshall S. A. (1982): A monograph of the genera Puncticorpus Duda, 1918 and Nearcticorpus gen.n. (Diptera, Sphaeroceridae). - Zool. Jahrb. Abt. Syst. Ökol. Geogr. Tiere 109: 357-398.

- (1986): The genus Trachyopella Duda (Diptera, Sphaeroceridae) of the Holarctic Region. Monografie III (1985), 109 pp., Museo Regionale di Scienze Naturali, Torino.

Roháček J., Marshall S. A., Norrbom A. L., Buck M., Quiros D. I. \& Smith I. (2001): World catalog of Sphaeroceridae (Roháček, J., ed.). 414 pp., Slezské zemské muzeum, Opava.

Roháček J. \& Papp. L. (2000): Crumomyia microps sp.n. from Austria and notes on other cavernicolous Crumomyia species (Diptera, Sphaeroceridae). - Annls Hist.-Nat. Mus. Natn. Hung. 92: 215-228.

Roháček J. \& Ševčík J. (2011): The fauna of Opetiidae and Platypezidae (Diptera) in the Gemer area (Central Slovakia). - Čas. Slez. Muz. Opava (A) 60: 41-47.

Ševčík J. (2010): Czech and Slovak Diptera associated with fungi. 112 pp., Slezské zemské muzeum. Opava.

Ševčík J. \& Kurina O. (2011): Fungus gnats (Diptera: Sciaroidea) of the Gemer region (central Slovakia): Part 1 - Bolitophilidae, Diadocidiidae, Ditomyiidae and Keroplatidae. - Čas. Slez. Muz. Opava (A) 60: 1123.

Thalhammer J. (1899): Ordo Diptera. In: Fauna regni Hungariae, III. Animalium Hungaricae hucusque cognitorum enumeratio systematica. Arthropoda (Insecta Diptera). 76 pp. Edidid regia societas scientiarum naturalium Hungarica, Akadémiai Kiadó, Budapest.

Troger H. \& Roháček J.(1980): Über die Sphaeroceridae-Fauna (Diptera) im Raum Obergurgl (Zentralalpen, Tirol): Faunistik, Taxonomie, Ökologie. - Beitr. Entomol. Berlin, 30: 15-33.

\section{Fauna čeledi Sphaeroceridae (Diptera) v Gemerské oblasti (střední Slovensko)}

Je podán přehled dvoukřídlých čeledi Sphaeroceridae (Diptera) zjištěných v Gemerské oblasti (Slovensko), která zahrnuje Národní parky Muránska planina, Slovenský raj a Slovenský kras. Na základě dříve publikovaných údajů a studovaného materiálu je z této oblasti uvedeno celkem 106 druhů. Každý z těchto druhů je pojednán s informacemi o jeho celkovém rozšíření, faunistice na Slovensku, bionomii a významu pro ochranu prŕrody. Regionální fauna Gemeru této čeledi je význačná nejen kvůli vysoké druhové rozmanitosti ale také proto, že zahrnuje četné velmi vzácné, stenotopní nebo ohrožené taxony, z nichž 5 druhů bylo z této oblasti popsáno, viz. Elachisoma bajzae Papp, 1983, Herniosina pollex Roháček, 1993, H. horrida (Roháček, 1978), Opalimosina (Opalimosina) calcarifera (Roháček, 1975) a Rachispoda segem (Roháček, 1991). K tomu je třeba zmínit i druhy, které jsou na Slovensku dosud známé pouze z území Gemeru, tedy Crumomyia setitibialis (Spuler, 1925), C. zuskai (Roháček, 1976), Spelobia faeroensis (Deeming, 1966) a S. simplicipes (Duda, 1925). Jsou diskutovány také další zvláštnosti a zajímavosti fauny čeledi Sphaeroceridae v Gemerské oblasti, včetně výčtu druhů zjištěných ve zdejších jeskynních systémech a přehledu nejvýznamnějších taxonů z hlediska ekologického, zoogeografického nebo pro ochranu př́írody na tomto území.

Author's address: Jindřich Roháček, Slezské zemské muzeum, Tyršova 1, CZ - 74601 Opava, Czech Republic; e-mail: rohacek@szmo.cz 